GPCRS

\section{Rules of attraction}

The directional response by motile cells to a chemical gradient, otherwise known as chemotaxis, is an important feature in many pathways, including inflammatory response. But although most chemotaxis pathways involve signal transduction mediated through Gprotein-coupled receptors (GPCRs), GPCRs in certain areas of the inflammatory response pathway have remained unidentified. Now, in Molecular Pharmacology, Carol Jones and colleagues at Novartis report the identification of a novel orphan GPCR that appears to mediate an important chemotactic response in immune cells.

Searches for additional members of the GPCR family in human genome databases led to the identification of R527. R527 has high similarity to the orphan receptors HM74, GPR81 and GPR31 at the amino-acid level, and was found to be highly expressed in immune cells, including eosinophils, neutrophils and lung macrophages. A cell line expressing R527 and the G protein $\alpha$-subunit (a promiscuous $\mathrm{G}$ protein which has been used to generate screening platforms for GPCR ligand fishing) was generated to screen a collection of 2,000 known and putative GPCR agonists. Leukotrienes, one class of chemotactic agents, failed to elicit a response, but two lipids activated

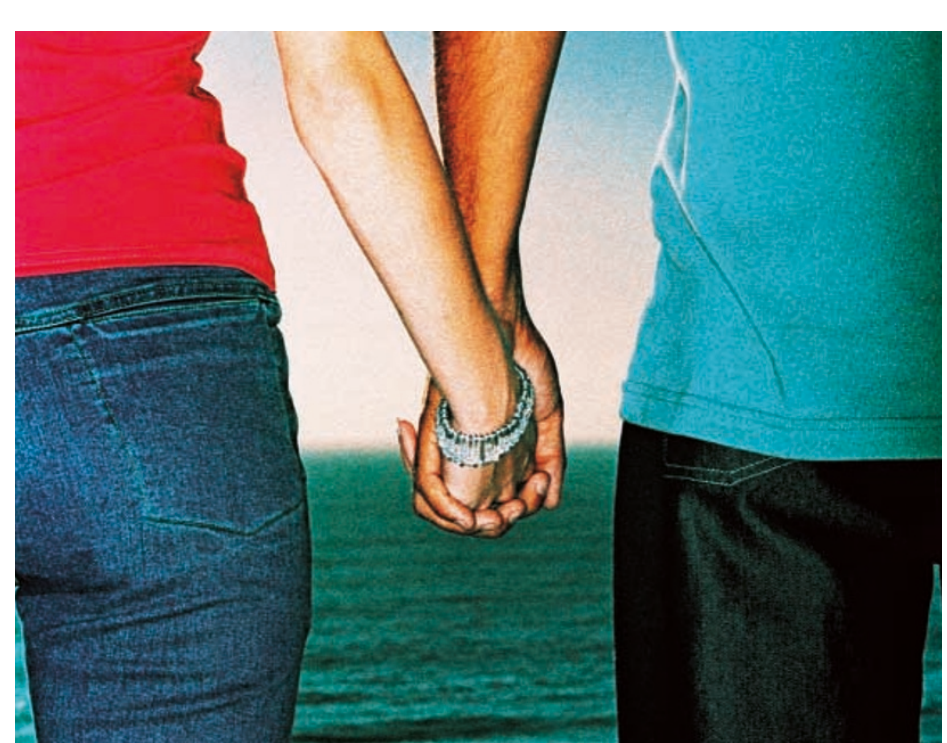

R527 - the most effective being the eicosanoid 5-oxo-6E, 8Z, 11Z, 14Zeicosatetraenoic acid (5-oxo-ETE). Increasing concentrations of 5-oxoETE added to the cell line inhibited the production of cyclic AMP, showing that R527 is coupled to the inhibitory subtype of $G$ proteins $\left(G \alpha_{i}\right)$, and this was confirmed by showing that pertussis toxin (which inhibits $G_{i}$ proteins) reverses this effect.

The findings could tie up some loose ends in this area. 5-oxo-ETE is known to be a potent simulator of chemotaxis and exerts its effects on eosinophils, neutrophils and monocytes. And the chemotactic responses induced by 5-oxo-ETE were thought to be mediated through a GPCR, the pharmacological properties of which appear to be similar to R527. Although it is possible that there might be other unidentified receptors that bind 5-oxo-ETE, evidence that 5-oxoETE could be a physiological mediator of inflammation in asthma indicates that R527 could have a key role in this and other inflammatory diseases (for example, psoriasis).

Simon Frantz

(D) References and links ORIGINAL RESEARCH PAPER Jones, C. E. etal. Expression and characterization of a 5-0xo-6E, $8 Z, 11 Z, 14 Z$-eicosatetraenoic acid receptor highly expressed on human eosinophils and neutrophils. Mol. Pharmacol. 63, 471-477 (2003)

\section{IN BRIEF}

\section{KINASE INHIBITORS}

Kinase inhibitors: not just for kinases anymore.

McGovern, S. L. \& Shoichet, B. K. J. Med. Chem. (2003) Mar 12 (doi:10.1021/jm020427b)

Small-molecule kinase inhibitors are widely used as tools for understanding the physiological roles of particular kinases and as leads for drug design; however, their use is often complicated by their lack of specificity. Although this can partly be accounted for by the similarity of the ATP-binding site between kinases, some kinase inhibitors also inhibit enzymes not known to bind ATP. The authors show that for several widely used kinase inhibitors, their nonspecificity could be due to the formation of aggregates, and so results obtained from the use of these compounds should be interpreted cautiously.

\section{ANTICANCER DRUGS}

Hedgehog signalling within airway epithelial progenitors and in small-cell lung cancer.

Watkins, D. N. et al. Nature 422, 313-316 (2003)

Small-cell lung cancer (SCLC) is highly aggressive and has very limited treatment options. Watkins et al. show that activation of the Hedgehog pathway, which has a wellcharacterized role in development, is involved in some types of SCLC, and that blockade of this pathway could be a novel therapeutic strategy.

\section{ANTI-OBESITY DRUGS}

Crystal structure of the carboxyltransferase domain of acetyl-coenzyme A carboxylase.

Zhang, H. et al. Science 299, 2064-2067 (2003)

Acetyl-coenzyme A carboxylases (ACCs), which regulate fatty acid biosynthesis, are a possible target for anti-obesity drugs. The carboxy-terminal (CT) domain of ACCs is also the target of several herbicides. Zhang et al. determined the structure of the CT domain of an ACC from yeast, and identified the probable area of the active site targeted by herbicides, thereby providing a starting point for the development of inhibitors of human ACCs.

\section{ANTICANCER DRUGS}

A tyrosine kinase created by fusion of the PDGFRA and FIP1L1 genes as a therapeutic target of imatinib in idiopathic hypereosinophilic syndrome.

Cools, J. et al. N. Engl J. Med. 348, 1201-1214 (2003)

Hypereosinophilic syndrome (HES) is a fatal blood disease that is characterized by an increase in inflammatory white blood cells. Cools et al. evaluated imatinib (Gleevec/Glivec; Novartis) in eleven patients with HES, nine of which had positive responses, and found evidence that the activity of imatinib could be due to inhibition of a tyrosine kinase produced by the fusion of the PDGFRA and FIPL1 genes. 